\title{
Improved QCD Sum-Rule Approach to Heavy-Meson Decay Constants
}

\author{
Wolfgang Lucha \\ Institute for High Energy Physics, Austrian Academy of Sciences, Nikolsdorfergasse 18, A-1050 \\ Vienna, Austria \\ E-mail: Wolfgang.Lucha@oeaw.ac.at

\section{Dmitri Melikhov*} \\ Institute for High Energy Physics, Austrian Academy of Sciences, Nikolsdorfergasse 18, A-1050 \\ Vienna, Austria, \\ Faculty of Physics, University of Vienna, Boltzmanngasse 5, A-1090 Vienna, Austria, and \\ D. V. Skobeltsyn Institute of Nuclear Physics, Moscow State University, 119991, Moscow, Russia \\ E-mail:dmitri_melikhov@gmx.de
}

\section{Silvano Simula}

INFN, Sezione di Roma Tre, Via della Vasca Navale 84, I-00146 Roma, Italy

E-mail: Simula@roma3.infn.it

\begin{abstract}
A visible improvement of the QCD sum-rule framework, achieved only recently by a slightly more sophisticated consideration of the hadron excitations and continuum, is applied to the extraction of the decay constants of heavy-light pseudoscalar mesons, such as the $D, D_{s}, B$ and $B_{s}$ mesons, from two-point correlators of heavy-light pseudoscalar currents [1]. This analysis is carried out entirely in terms of the running heavy-quark mass, for which the perturbative expansion exhibits a passable convergence. Our main concern is to gain control over both statistical and systematic uncertainties of the extracted decay constants, the former being induced by the QCD parameter values requested for input, the latter arising from the intrinsically limited accuracy of the QCD sum-rule techniques.
\end{abstract}

The 2011 Europhysics Conference on High Energy Physics, EPS-HEP 2011,

July 21-27, 2011

Grenoble, Rhône-Alpes, France

* Speaker. 


\section{Introduction}

Although the QCD sum-rule framework has been proposed even more than thirty years ago [2], within this approach any study of the decay constant $f_{P}$ of a ground-state heavy pseudoscalar meson $P=(Q \bar{q})$ of mass $M_{P}$, viewed as bound state of a heavy quark $Q$ and a light antiquark $\bar{q}$ with masses $m_{Q}$ and $m$, respectively, still forms a nontrivial problem, for, at least, two easily identifiable reasons:

- A trustable operator product expansion (OPE) for the vacuum two-point correlation function,

$$
\Pi\left(p^{2}\right) \equiv \mathrm{i} \int \mathrm{d}^{4} x \exp (\mathrm{i} p x)\left\langle 0\left|\mathrm{~T}\left(j_{5}(x) j_{5}^{\dagger}(0)\right)\right| 0\right\rangle,
$$

of two pseudoscalar heavy-light currents $j_{5}(x)=\left(m_{Q}+m\right) \bar{q}(x)$ i $\gamma_{5} Q(x)$ must be constructed.

- For obvious practical reasons, the OPE is known only in some truncated form; this means that even if its parameters were known precisely, this limitation prevents to extract the bound-state features with arbitrarily high accuracy and betrays an intrinsic uncertainty of QCD sum rules.

A crucial result is that assuming quark-hadron duality relates the lowest bound-state contribution to the OPE for a Borel-transformed correlator $\Pi_{\text {dual }}\left(\tau, s_{\text {eff }}\right)$ cut at an effective continuum threshold $s_{\text {eff: }}$ :

$$
f_{P}^{2} M_{P}^{4} \exp \left(-M_{P}^{2} \tau\right)=\Pi_{\text {dual }}\left(\tau, s_{\text {eff }}\right) \equiv \int_{\left(m_{Q}+m\right)^{2}}^{s_{\text {eff }}} \mathrm{d} s \exp (-s \tau) \rho_{\text {pert }}(s)+\Pi_{\text {power }}(\tau)
$$

with perturbative spectral density $\rho_{\text {pert }}(s)$, expressible as series expansion in the strong coupling $\alpha_{\mathrm{s}}$. Before being able to extract hadron features, we have to know the effective continuum threshold $s_{\text {eff }}$. The decay constant $f_{P}$ extracted from (1.2) turns out to depend on the unphysical Borel parameter $\tau$; the standard - but, from our point of view, maybe too naïve - guess for a, by assumption constant, $s_{\text {eff }}$ is found by minimizing the $\tau$-dependence of $f_{P}$. However, an analogy to quantum-mechanics [3] reveals that this does not guarantee the reliability of the $f_{P}$ prediction. The reason for this fact is that any exact effective continuum threshold, found by solving Eq. (1.2) for known values of $M_{P}$ and $f_{P}$, will, in general, depend on $\tau$. (In quantum mechanics, the true bound-state masses and decay constants are found from the solutions of the Schrödinger equation.) Of course, the exact effective continuum threshold is not known. Thus, any sum-rule extraction of hadronic features consists in attempting to arrive somehow at a reasonable approximation to the true effective continuum threshold, and to gain control over the accuracy of this approximation; the relevant techniques have been developed in [ $[$ 由.

A brief glance at the shape of the hadronic ground-state contribution on the left-hand side of the sum rule (1.2) prompts us to introduce a dual invariant mass $M_{\text {dual }}$ and a dual decay constant $f_{\text {dual }}$ by

$$
M_{\text {dual }}^{2}(\tau) \equiv-\frac{\mathrm{d}}{\mathrm{d} \tau} \log \Pi_{\text {dual }}\left(\tau, s_{\mathrm{eff}}(\tau)\right), \quad f_{\text {dual }}^{2}(\tau) \equiv M_{P}^{-4} \exp \left(M_{P}^{2} \tau\right) \Pi_{\text {dual }}\left(\tau, s_{\text {eff }}(\tau)\right) .
$$

For known true ground-state mass, the deviation of the dual mass $M_{\text {dual }}$ from the actual ground-state mass $M_{P}$ must be considered as indication of the amount of excited-state contributions picked up by the dual correlator defined by Eq. 1.2. . Assuming for the effective continuum threshold a particular functional form and requiring least deviation of the dual mass (1.3) from its actual value in the range of admissible $\tau$-values yields a variational solution for the effective threshold. With the latter fixed, the decay constant follows from (1.3). Allowing for this $\tau$-dependence of our threshold facilitates to reproduce the actual mass and improves the accuracy of extracted hadron observables considerably. 


\section{Operator Product Expansion and Heavy-Quark Mass Scheme}

The choice of the precise scheme employed for the definition of the masses of the heavy quarks is crucial in analyses of the present kind. The correlator (1.1) has been derived to three-loop order in terms of the heavy quark's pole mass [5], which has been standard for a long time [6]. Alternatively, the perturbative expansion may be reorganized in terms of the running $\overline{\mathrm{MS}}$ mass [7]. Figure 1 shows our dual sum-rule estimates for $f_{B}$ resulting for both cases. These plots allow for important insights:

1. The perturbative expansion for $f_{\text {dual }}$ in terms of the pole mass reveals no sign of convergence: LO, NLO, and NNLO terms contribute with similar size and higher orders cannot be expected to give smaller contributions. Therefore, the pole-mass OPE considerably underestimates $f_{B}$.

2. As already noticed in [D], reorganizing the perturbative expansion in terms of the running $\overline{\mathrm{MS}}$ mass of the heavy quark yields a distinct hierarchy of the perturbative contributions. It should be no surprise that we prefer the OPE in terms of the $\overline{\mathrm{MS}}$ mass in our decay-constant analysis.

3. The magnitude of $f_{\text {dual }}$ extracted from the pole-mass OPE is almost $50 \%$ smaller than that one found within the $\overline{\mathrm{MS}}$ scheme; nevertheless, in both cases the decay constants exhibit a perfect stability in a wide range of the Borel parameter $\tau$. Thus, we conclude that mere Borel stability does not suffice to guarantee the reliability of any sum-rule extraction of bound-state features!
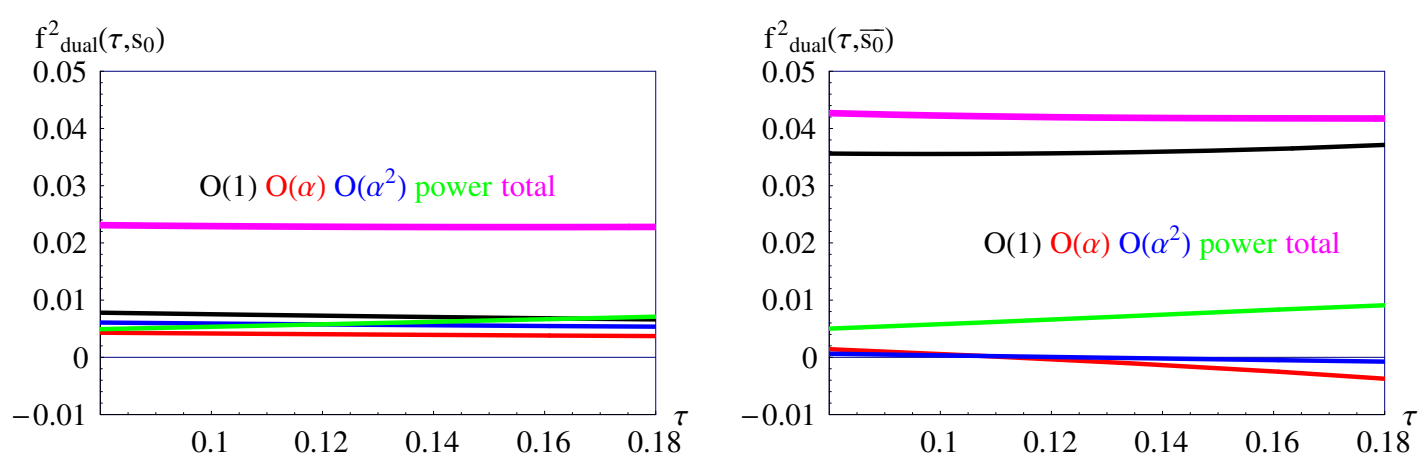

Figure 1: Dual estimates $f_{\text {dual }}$ for the $B$-meson decay constant $f_{B}$ extracted from the OPE expressed in terms of pole mass (left) and $\overline{\mathrm{MS}}$ mass (right) of the $b$ quark. By requesting maximal stability of the extracted $f_{\text {dual }}$, in each case a constant effective threshold $\left(\overrightarrow{s_{0}}\right)$ is determined individually, thus $s_{0}$ and $\overline{s_{0}}$ differ from each other. The relative power-correction contributions rise with $\tau$. All quantities in units of appropriate powers of GeV.

\section{Decay Constants of Open-Charm $D$ and $D_{s}$ Mesons}

Our analysis of the decay constants of the $D$ mesons [1] clearly demonstrates that the improved QCD sum-rule approach [ $₫$ relying on effective continuum thresholds which we allow to depend on the Borel parameter $\tau$ yields a much better accuracy of the quark-hadron duality approximation and a distinct improvement of the accuracy of the extracted decay constants. This perfectly confirms our observations [8] (respectively, conviction) that in QCD and in quantum mechanics the procedures of extracting bound-state characteristics are, qualitatively as well as quantitatively, very similar to each other. Moreover, all estimates of systematic errors given by our algorithm prove to be quite realistic. 


\section{Decay Constants of Open-Beauty $B$ and $B_{s}$ Mesons and Running $b$-Quark Mass}

The predictions of QCD sum rules for the $B$-meson decay constants $f_{B}$ and $f_{B_{s}}$, analyzed in due detail in [1], prove to be extremely sensitive to the precise $\bar{m}_{b}\left(\bar{m}_{b}\right)$ value used as input. For instance, the choice $\bar{m}_{b}\left(\bar{m}_{b}\right)=(4.163 \pm 0.016) \mathrm{GeV}$ ["] entails decay-constant predictions hardly compatible with lattice findings. However, inverting the logic by fitting our sum-rule result for $f_{B}$ to the average of lattice findings yields the very precise value of the $b$-quark mass $\bar{m}_{b}\left(\bar{m}_{b}\right)=(4.245 \pm 0.025) \mathrm{GeV}$.

\section{Summary, Observations, and Conclusions}

1. The $\tau$-dependence of effective thresholds arises quite naturally by our attempts to increase the precision of the duality concept; relaxing the limitation to constant thresholds distinctly raises both the stability of the dual mass and the quality of sum-rule predictions for decay constants.

2. Our study of charmed mesons clearly demonstrates that adopting Borel-parameter-dependent thresholds significantly improves the accuracy of extractions of decay constants by sum rules, yields meaningful systematic errors, reduces these to the level of a few percent, and brings the sum-rule results into perfect agreement with the findings of both lattice QCD and experiment.

3. The decay constants of beauty mesons are unexpectedly sensitive to the chosen $\bar{m}_{b}\left(\bar{m}_{b}\right)$ value. We may, however, regard this inconvenience as a kind of serendipity that allows us to derive a rather accurate estimate of the $b$-quark mass by matching the QCD sum-rule prediction for $f_{B}$ to the average of the corresponding lattice evaluations. The value of $m_{b}$ deduced in this way is in good agreement with lattice findings but lacks overlap with a recent accurate determination of $m_{b}$ [9] (for details, consult [1]); beyond doubt, this disconcerting puzzle has to be resolved.

Acknowledgments. D.M. was supported by the Austrian Science Fund (FWF), project no. P22843.

\section{References}

[1] W. Lucha, D. Melikhov, and S. Simula, J. Phys. G 38 (2011) 105002; Phys. Lett. B 701 (2011) 82.

[2] M. Shifman, A. Vainshtein, and V. Zakharov, Nucl. Phys. B 147 (1979) 385.

[3] W. Lucha, D. Melikhov, and S. Simula, Phys. Rev. D 76 (2007) 036002; Phys. Lett. B 657 (2007) 148; Phys. Atom. Nucl. 71 (2008) 1461; Phys. Lett. B 671 (2009) 445; D. Melikhov, Phys. Lett. B 671 (2009) 450.

[4] W. Lucha, D. Melikhov, and S. Simula, Phys. Rev. D 79 (2009) 096011; J. Phys. G 37 (2010) 035003; W. Lucha, D. Melikhov, H. Sazdjian, and S. Simula, Phys. Rev. D 80 (2009) 114028.

[5] K. G. Chetyrkin and M. Steinhauser, Phys. Lett. B 502 (2001) 104; Eur. Phys. J. C 21 (2001) 319.

[6] T. M. Aliev and V. L. Eletsky, Yad. Fiz. 38 (1983) 1537.

[7] M. Jamin and B. O. Lange, Phys. Rev. D 65 (2002) 056005.

[8] W. Lucha, D. Melikhov, and S. Simula, Phys. Lett. B 687 (2010) 48; Phys. Atom. Nucl. 73 (2010) 1770.

[9] K. G. Chetyrkin et al., Phys. Rev. D 80 (2009) 074010. 